Brazilian Journal

of Chemical

Engineering

\title{
SIMULATION OF THERMAL DECOMPOSITION IN AN OPEN CAVITY: ENTROPY ANALYSIS
}

\author{
Guillermo E. Ovando-Chacon ${ }^{1 *}$, Sandy L. Ovando-Chacon², Juan C. Prince-Avelino', \\ Abelardo Rodriguez-Leon ${ }^{1}$ and Cesar Garcia-Arellano ${ }^{1,3}$
}

\author{
${ }^{1}$ Instituto Tecnológico de Veracruz, Tecnológico Nacional de México, Departamento Metal-Mecánica y Mecatrónica, \\ Veracruz, México. E-mail: ovachag@hotmail.com, ORCID: 0000-0003-2441-3021; ORCID: 0000-0002-1056-2272; \\ ORCID: 0000-0002-2179-4837; ORCID: 0000-0003-2778-8355 \\ ${ }^{2}$ Instituto Tecnológico de Tuxtla Gutiérrez, Tecnológico Nacional de México, Departamento Química y Bioquímica, \\ Chis, México. ORCID: 0000-0001-8754-0269 \\ ${ }^{3}$ Universidad Papaloapan, Campus Loma Bonita, Instituto de Agroingeniería, Loma Bonita, Mèxico.
}

(Submitted: July 14, 2017 ; Revised: January 6, 2018 ; Accepted: March 15, 2018)

\begin{abstract}
This paper presents a numerical analysis of entropy generation in a two-dimensional rectangular channel where the inlet flow undergoes thermal decomposition resulting from a chemical reaction. The model considered viscosity and thermal conductivity to be dependent of temperature. Irreversibility due to mass transport was included in the entropy generation analysis. Relevant applications of this study are possible for the design of power generation systems and reactors. The effects of the Reynolds number, Schmidt number, and length of the heat source on thermal fluid dynamics, mass transfer, and irreversibility were also investigated. It was found that thermal decomposition increases at: a) low Reynolds numbers, b) low Schmidt numbers, and c) increased length of heat source. Additionally, overall entropy generation increased when Reynolds number and length of heat source were increased, although in all cases, overall irreversibility attains a minimum value at a specific Schmidt number.

Keywords: Entropy generation; Thermal decomposition; Finite element; Chemical reaction.
\end{abstract}

\section{INTRODUCTION}

Over the past decades, flow simulations in open systems have received considerable attention due to their relevance for a number of engineering, industrial, and natural applications; however, there is a lack of research related to hydrodynamics and entropy generation in systems where chemical reactions take place in the fluid. Examples of practical applications of the current research in the field are reactor and energy conversion systems: Bousri et al. (2017) performed simulations of heat and mass transfer in a system involving a chemical reaction in a two-dimensional channel with impermeable walls and uniform heat flux. The flow was assumed to be steady and incompressible. Harfash and Alshara (2015) analyzed the effect of chemical reactions on a double diffusive convection model in a porous cavity, and presented the stability analysis and numerical simulations of the convective motion of an incompressible fluid. Lazarovici et al. (2005) carried out a numerical investigation of the convection within a square reactor modeled as a closed cavity with chemical reactions. The governing equations for an incompressible flow were solved by the vorticity-stream function approach. Luo et al. (2004) discussed the results of computations aimed toward an acceptable scaled-up version of an impinging jet chemical reactor modeled as an open cavity. They were successful in determining geometry and a range of appropriate flow parameters by

\footnotetext{
* Corresponding author: Guillermo E. Ovando-Chacon - E-mail: ovachag@hotmail.com
} 
analyzing streamline, concentration, and temperature contours. Van Saten et al. (2001) conducted another numerical study of laminar flow and heat transfer in a chemical decomposition reactor. They analyzed the convection regime, recirculation cells, temperature contours, and Nusselt number. Waghode et al. (2005) reported on the simulation of fluid dynamics and heat transfer in a microreactor modeled as an open cavity and obtained data for velocity, pressure, concentration, and temperature fields. Lin et al. (2009) considered a two-dimensional steady laminar flow with heat and mass transfer, as well as wall surface reaction, in a vertical reactor, and calculated streamlines, isotherms, and deposition rate. All these studies indicate that the configuration of the cavity and the properties of the fluid affect the thermal fluid dynamics of the flow.

On the other hand, the search for the conditions that control heat and mass transfer in systems without chemical reactions modeled as cavities has received considerable attention from many researchers. Specifically, open configurations have been numerically studied by several authors. Mariani and Coelho (2007) investigated the natural convection due to the temperature differences between left and right walls and an internal heat source in open enclosures. Rahman et al. (2013) carried out an investigation of a two-dimensional laminar incompressible mixed convection flow in a ventilated cavity. Fontana et al. (2016) carried out a comparison between linear stability analysis and numerical simulation of Rayleigh-Benard convection in double-layer channel flow. Dhiman et al. (2012) analyzed the effects of blockage ratio on the cross-buoyancy around a confined square cylinder in a channel. Zamora and Kaiser (2016, 2014) studied natural convection and radiation in open cavities taking into account the variable thermophysical properties of air.

Finally, entropy generation analyses to determine optimal design criteria have recently become a topic of interest in many engineering and industrial fields. What follows is a review of the entropy generation literature. Zehtabiyan-Rezaie et al. (2017) performed a numerical study of heat transfer enhancement and entropy generation in the flow inside open cavities in the presence of inclined magnetic fields. Khan and Gorla (2012) presented the second law analysis of heat transfer and fluid flow due to free convection over a horizontal plate with prescribed surface heat flux. Shuja et al. (2008) studied flow over a porous block inside a channel and numerically studied the effects of channel inlet port height, porosity, and block aspect ratio on entropy generation. Cheng and Huang (1989) numerically evaluated the entropy generated by a laminar two-dimensional force convection flow over transverse fins attached to a horizontal channel. Chen et al. (2014) reported numerical studies on heat transfer performance and entropy generation rate in a vertical channel containing a fully developed flow of nanofluid. Bermejo et al. (2013) carried out the process of minimization of the total entropy generation in an evaporator and obtained an expression to estimate the best channel width that minimizes the entropy generation. Mahmud and Fraser (2005) examined the flow, thermal and entropy generation fields inside a parallel plate channel and analyzed the velocity and temperature profiles, the Bejan number and the average entropy generation number.

The previous literature review shows that fluid dynamics, heat, and mass transport in open and closed systems have been widely studied; however, to the best of our knowledge, no attention has been paid to thermal characterization in channels where chemical reactions are taking place. The main objective of the present study is to analyze the effects of Reynolds number, Schmidt number, and length of the heat source on the generation of entropy in flow inside an open rectangular system due to thermal decomposition driven by a chemical reaction. The main contribution in the present study is the characterization of irreversibility production due to complex interactions between fluid dynamics and heat and mass transfer inside an open cavity where the fluid undergoes thermal decomposition; the results may have potential applications in the design of chemical and nuclear reactors and energy conversion devices (Bousri et al. (2017), Harfash and Alshara (2015), Lazarovici et al. (2005), Luo et al. (2004), Van Saten et al. (2001), Waghode et al. (2005), Lin et al. (2009)).

The originality of this work consists of numerically analyzing the generation of entropy, through the hydrodynamic characterization of complex nonlinear phenomena that arise in convection-diffusion processes coupled to a chemical reaction, which has not been addressed by other researchers. The study of entropy generation is justified because, by minimizing entropy, the operating parameters are known with which a device can operate with the minimum energy consumption, contributing to the efficient use of energy and the environment care.

\section{FORMULATION OF THE PROBLEM}

The model analyzed in this study consists of a twodimensional rectangular open cavity with a chemical reaction that produces thermal decomposition, see Figure 1. The cavity is open at the upper left wall and at the lower right wall, where the fluid respectively enters and leaves the system. A hot plate is located at the center of the bottom wall; different lengths of the heat source $\mathrm{Wp}=0.2$ to 0.4 were modeled. In order to investigate the effect of the viscous diffusion rate against the molecular diffusion rate, the Schmidt number Sc was varied from 1 to 100 . The effect 


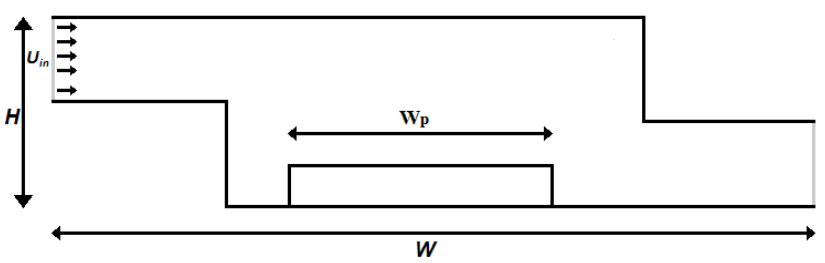

Figure 1. Sketch of the computational domain used to study the heat transfer and entropy generation in an open system.

of fluid dynamics on thermal decomposition was analyzed by Reynolds number Re, which was varied from 100 to 1000. Zero concentration gradient, no slip, and adiabatic boundary conditions were imposed on the walls of the domain, except for the place where the heat source was located. The relevant dimensionless boundary conditions imposed on the cavity were as follows: inlet flow temperature was fixed to $\mathrm{T}=0$; isothermal hot plate temperature was fixed to $\mathrm{T}_{\mathrm{h}}^{\mathrm{c}}=1$; and a high concentration $\mathrm{C}_{\mathrm{H}}=1$ was imposed at the entrance of the cavity.

The problem described above may have applications in chemical reactions with a single reactant, that is, unimolecular reactions where heat is applied to decompose into a product $\mathrm{A}$ and a product $\mathrm{B}$, one of these chemical reactions being the thermal decomposition of ammonia $\mathrm{NH}_{3}$, which decomposes into nitrogen $\mathrm{N}_{2}$ and hydrogen $\mathrm{H}_{2}$. In this sense, the present investigation can be applied to obtaining $\mathrm{H}_{2}$ for its use in fuel cells (Waghode et al. (2005), Cheddie (2012)) contributing to the environment by reducing pollutants to the atmosphere.

\section{MATHEMATICAL MODEL}

\section{Transport Equations}

The study of a flow through a channel with heat and mass transfer requires the solution of the Navier-Stokes equations (continuity and momentum conservation), coupled to the energy and mass transport conservation equations, that have been widely reported in the literature (Anderson (1995), Zienkiewicz (2014)).

The dimensionless Navier-Stokes equations, together with the mass and energy equations which govern an incompressible fluid with heat and mass transfer due to a chemical reaction in a twodimensional domain $\Omega$ can be written using the following dimensionless variables:

$$
\begin{array}{llll}
\mathrm{x}=\frac{\mathrm{x}^{\prime}}{\mathrm{W}} \quad \mathrm{y}=\frac{\mathrm{y}^{\prime}}{\mathrm{W}} \quad \mathrm{t}=\frac{\mathrm{U}_{\text {in }} \mathrm{t}^{\prime}}{\mathrm{W}} \quad \mathrm{u}_{1}=\frac{\mathrm{u}_{1}^{\prime}}{\mathrm{U}_{\text {in }}} \\
\mathrm{u}_{2}=\frac{\mathrm{u}_{2}^{\prime}}{\mathrm{U}_{\text {in }}} \quad \mathrm{p}=\frac{\mathrm{p}}{\rho \mathrm{u}_{\text {in }}^{2}} \quad \mathrm{~T}=\frac{\mathrm{T}^{\prime}-\mathrm{T}_{\mathrm{c}}^{\prime}}{\mathrm{T}_{\mathrm{h}}^{\prime}-\mathrm{T}_{\mathrm{c}}^{\prime}} \quad \mathrm{C}=\frac{\mathrm{C}^{\prime}}{\mathrm{C}_{\mathrm{H}}^{\prime}}
\end{array}
$$

$$
\begin{aligned}
& \frac{\partial \mathrm{u}}{\partial \mathrm{t}}+\mathrm{u} \cdot \nabla \mathrm{u}-\frac{1}{\operatorname{Re}}[\nabla \cdot(\mu \nabla \mathrm{u})]+\nabla \mathrm{p}=\operatorname{RiT} \hat{\mathrm{J}} \text { in } \Omega \times\left[0, \mathrm{t}_{\mathrm{f}}\right] \\
& \nabla \cdot \mathrm{u}=0 \text { in } \Omega \times\left[0, \mathrm{t}_{\mathrm{f}}\right] \\
& \frac{\partial \mathrm{T}}{\partial \mathrm{t}}+\mathrm{u} \cdot \nabla \mathrm{T}-\frac{1}{\operatorname{ReSc}}[\nabla \cdot(\mathrm{k} \nabla \mathrm{T})]=\mathrm{Q} \text { in } \Omega \times\left[0, \mathrm{t}_{\mathrm{f}}\right] \\
& \frac{\partial \mathrm{C}}{\partial \mathrm{t}}+\mathrm{u} \cdot \nabla \mathrm{C}-\frac{1}{\operatorname{ReSc}}[\nabla \cdot(\nabla \mathrm{C})]=\mathrm{R}_{\mathrm{mt}} \text { in } \Omega \times\left[0, \mathrm{t}_{\mathrm{f}}\right]
\end{aligned}
$$

The velocity vector is given by $\mathrm{u}=\left(\mathrm{u}_{1}, \mathrm{u}_{2}\right)$, where $\mathrm{u}_{1}$ is the axial component and $\mathrm{u}_{2}$ is the transversal component, $\mathrm{Pr}$ is the Prandtl number, $\mathrm{p}$ is pressure, $\mathrm{t}$ is time, $\mu$ is dynamic viscosity, $\mathrm{t}_{\mathrm{f}}$ is final time, which is the time where steady state is reached, $\mathrm{T}$ is temperature, $\mathrm{k}$ is thermal conductivity, and $\mathrm{C}$ is concentration. The source terms for the energy and mass equations are respectively given by $\mathrm{Q}$ and $\mathrm{R}_{\mathrm{mt}}$. Schmidt number, Reynolds number, Prandtl number, and Richardson number can be written as:

$$
\begin{array}{ll}
\mathrm{Sc}=\mu_{\mathrm{ref}} / \rho \mathrm{D} & \operatorname{Pr}=\mu_{\mathrm{ref}} \mathrm{C}_{\mathrm{p}} / \mathrm{k}_{\text {ref }} \\
\mathrm{Re}=\rho \mathrm{WU} \mathrm{Hn}_{\text {in }} / \mu_{\text {ref }} & \mathrm{Ri}=\beta \mathrm{Wg}\left(\mathrm{T}_{\mathrm{h}}^{\prime}-\mathrm{T}_{\mathrm{c}}^{\prime}\right) / \mathrm{U}_{\text {in }}^{2}
\end{array}
$$

In this study, the Richardson number was fixed to $\mathrm{Ri}=1$, this value was selected based on the result reported by Luo et al. (2004), who determined that the optimum design of a reactor occurs when $\mathrm{Ri}=1.191$, a value close to unity where the effects of forced and natural convection are equal. $U_{\text {in }}$ is the velocity of the flow at the inlet, $\mathrm{D}$ is the diffusion coefficient, $\rho$ is density, and $\mathrm{W}$ is cavity length. Specific heat capacity, reference dynamic viscosity, and reference thermal conductivity are given by $\mathrm{C}_{\mathrm{p}}, \mu_{\mathrm{ref}}$, and $\mathrm{k}_{\mathrm{ref}}$, respectively. Additionally, $\mathrm{R}_{\mathrm{mt}}$ and $\mathrm{Q}$ can be written as:

$\mathrm{R}_{\mathrm{mt}}=\frac{-\mathrm{Wr}}{\mathrm{U}_{\mathrm{in}} \mathrm{C}_{\mathrm{H}}^{\prime}}$

and

$\mathrm{Q}=\frac{\Phi r \mathrm{~W}}{\mathrm{C}_{\mathrm{p}} \mathrm{U}_{\mathrm{in}} \rho\left(\mathrm{T}_{\mathrm{h}}^{\prime}-\mathrm{T}_{\mathrm{c}}^{\prime}\right)}$

where reaction heat is given by $\Phi$ and reaction rate is given by $r$ :

$$
\mathrm{r}=\gamma \mathrm{C}^{\prime}
$$


here $\gamma$ is the rate constant:

$$
\gamma=\mathrm{Ae}^{-\mathrm{E} / \mathrm{R}_{\mathrm{g}} \mathrm{T}^{\prime}}
$$

where $\mathrm{A}$ is the pre-exponential factor, $\mathrm{E}$ is the activation energy and $R_{g}$ is the gas constant. It is important to note that $\mathrm{Q}$ and $\mathrm{R}_{\mathrm{mt}}$ depend on temperature through the reaction rate $r$, which is a function of the rate constant $\gamma$, which in turn depends on the temperature.

The dimensionless initial conditions assumed throughout the cavity were fluid at rest, uniform temperature $\mathrm{T}=0$, and uniform concentration $\mathrm{C}=0$. The variations of thermal conductivity and viscosity as a function of temperature were included in the model as follows:

$$
\begin{aligned}
& \mathrm{k}^{\prime}=1-0.7688 \cdot\left(\mathrm{T}^{\prime}-1\right)+1.1677\left(\mathrm{~T}^{\prime}-1\right)^{2} \\
& \mu^{\prime}=1-5.6386 \cdot\left(\mathrm{T}^{\prime}-1\right)+12.2476\left(\mathrm{~T}^{\prime}-1\right)^{2}
\end{aligned}
$$

The previous relationships were obtained considering $\mathrm{NH}_{3}$ (Cengel, 2007) and the dimensionless forms are obtained as:

$$
\mathrm{k}=\frac{\mathrm{k}^{\prime}}{\mathrm{k}_{\mathrm{ref}}} \text { and } \mu=\frac{\mu^{\prime}}{\mu_{\mathrm{ref}}}
$$

\section{Entropy Generation Equations}

To calculate the entropy generation in fluid flow problems with heat and mass transfer, it is necessary to obtain the velocity, temperature and concentration gradients from their respective distributions obtained by solving the conservation equations. Next, the equations are described for calculating entropy generation due to viscous dissipation, thermal diffusion and concentration diffusion that have been used in several research works (De Groot and Mazur (1984), Ghachem et al. (2012)).

Total dimensionless local entropy generation in a flow with mass transfer can be written as:

$$
\begin{aligned}
\mathrm{S}_{1} & =\varnothing\left[2\left(\frac{\partial \mathrm{u}_{1}}{\partial \mathrm{x}}\right)^{2}+2\left(\frac{\partial \mathrm{u}_{2}}{\partial \mathrm{y}}\right)^{2}+\left(\frac{\partial \mathrm{u}_{1}}{\partial \mathrm{y}}+\frac{\partial \mathrm{u}_{2}}{\partial \mathrm{x}}\right)^{2}\right]+ \\
& +\left[\left(\frac{\partial \mathrm{T}}{\partial \mathrm{x}}\right)^{2}+\left(\frac{\partial \mathrm{T}}{\partial \mathrm{y}}\right)^{2}\right]+\varnothing_{\mathrm{mt}}\left[\left(\frac{\partial \mathrm{C}}{\partial \mathrm{x}}\right)^{2}+\left(\frac{\partial \mathrm{C}}{\partial \mathrm{y}}\right)^{2}\right]+ \\
& +\varnothing_{\mathrm{mt} t}\left[\left(\frac{\partial \mathrm{T}}{\partial \mathrm{x}}\right)\left(\frac{\partial \mathrm{C}}{\partial \mathrm{x}}\right)+\left(\frac{\partial \mathrm{T}}{\partial \mathrm{y}}\right)\left(\frac{\partial \mathrm{C}}{\partial \mathrm{y}}\right)\right]
\end{aligned}
$$

The first term is the local generation of entropy due to fluid friction:

$$
\mathrm{S}_{\mathrm{ffl}}=\varnothing\left[2\left(\frac{\partial \mathrm{u}_{1}}{\partial \mathrm{x}}\right)^{2}+2\left(\frac{\partial \mathrm{u}_{2}}{\partial \mathrm{y}}\right)^{2}+\left(\frac{\partial \mathrm{u}_{1}}{\partial \mathrm{y}}+\frac{\partial \mathrm{u}_{2}}{\partial \mathrm{x}}\right)^{2}\right]
$$

The second term is the local generation of entropy due to heat transfer:

$$
\mathrm{S}_{\mathrm{htl}}=\left[\left(\frac{\partial \mathrm{T}}{\partial \mathrm{x}}\right)^{2}+\left(\frac{\partial \mathrm{T}}{\partial \mathrm{y}}\right)^{2}\right]
$$

The third term is the local generation of entropy due to mass transfer:

$$
\begin{aligned}
\mathrm{S}_{\mathrm{mtl}} & =\varnothing_{\mathrm{mt}}\left[\left(\frac{\partial \mathrm{C}}{\partial \mathrm{x}}\right)^{2}+\left(\frac{\partial \mathrm{C}}{\partial \mathrm{y}}\right)^{2}\right]+ \\
& +\varnothing_{\mathrm{mht}}\left[\left(\frac{\partial \mathrm{T}}{\partial \mathrm{x}}\right)\left(\frac{\partial \mathrm{C}}{\partial \mathrm{x}}\right)+\left(\frac{\partial \mathrm{T}}{\partial \mathrm{y}}\right)\left(\frac{\partial \mathrm{C}}{\partial \mathrm{y}}\right)\right]
\end{aligned}
$$
follows:

Overall generation of entropy is obtained as

$$
\mathrm{S}=\int_{\Omega} \mathrm{S}_{\mathrm{htl}} \mathrm{dA}+\int_{\Omega} \mathrm{S}_{\mathrm{ffl}} \mathrm{dA}+\int_{\Omega} \mathrm{S}_{\mathrm{mtl}} \mathrm{dA}
$$

The ratio of global heat and mass transfer irreversibilities to overall entropy production is the average Bejan number:

$\mathrm{Be}=\frac{\mathrm{S}_{\mathrm{ht}}+\mathrm{S}_{\mathrm{mt}}}{\mathrm{S}}$

The Bejan number is important because it allows measuring the contribution of irreversibilities due to temperature and concentration gradients with respect to the total generation of entropy, in a way that allows determining the type of dominant irreversibility in a device or process. Equation (19) is based on both the irreversibilities due to the temperature $\mathrm{S}_{\mathrm{ht}}$ and the irreversibilities due to the concentration $\mathrm{S}_{\mathrm{mt}}$, which assures that the concentration gradients are included. This definition of Bejan's number has been used in recent works (Chen et al. (2015), Ghachem (2012)). The reason for making this modification in the Bejan number is that the entropy due to the concentration $\mathrm{S}_{\mathrm{mt}}$ has two components; as indicated by Eq. 17, the first contains only concentration gradients and the second contains combined gradients of concentration and temperature. Therefore, irreversibilities due to concentration are strongly associated with temperature gradients. 


\section{COMPUTATIONAL IMPLEMENTATION}

The system of strongly coupled governing equations defined by Eqs. (2)-(5), combined with the boundary conditions stated earlier, was solved using a stabilized finite element method, which guaranteed the elimination of instabilities and spurious oscillations of the convection-diffusion reaction equation. The stabilized finite element method allows an accurate evaluation of the necessary velocity, concentration, and temperature gradients to accurately estimate entropy generation; detailed information about this method can be found in recent literature and this will not be repeated here, see for example Volker et al. (2009), Volker et al. (2008), Hauke et al. (2007) and Duan et al. (2014). The governing equations are split into seven sub-problems using a splitting scheme (Ganesan and Tobiska (2012), Ganesan (2012)) given by:

$$
\begin{aligned}
& \int_{\Omega} \frac{\mathrm{u}^{\mathrm{n}+1 / 3-\mathrm{u}^{\mathrm{n}}}}{\Delta \mathrm{t}} \cdot \sigma \mathrm{dX}+\int_{\Omega} \mathrm{p}^{\mathrm{n}+1 / 3} \nabla \cdot \sigma \mathrm{dX}=0 \\
& \int_{\Omega} \sigma \nabla \mathrm{u}^{\mathrm{n}+1 / 3} \mathrm{dX}=0 \\
& \int_{\Omega} \frac{\mathrm{u}^{\mathrm{n}+2 / 3-\mathrm{u}^{\mathrm{n}+1 / 3}}}{\Delta \mathrm{t}} \cdot \sigma \mathrm{dX}+\int_{\Omega}\left(\mathrm{u}^{\mathrm{n}+1 / 3} \cdot \nabla\right) \mathrm{u} \cdot \sigma \mathrm{dX}=0
\end{aligned}
$$

$$
\int_{\Omega} \frac{\mathrm{T}^{\mathrm{n}+2 / 3-\mathrm{T}^{\mathrm{n}}}}{\Delta \mathrm{t}} \cdot \sigma \mathrm{dX}+\int_{\Omega}\left(\mathrm{u}^{\mathrm{n}+1 / 3} \cdot \nabla\right) \mathrm{T}^{\mathrm{n}+2 / 3} \cdot \sigma \mathrm{dX}=0
$$

$\int_{\Omega} \frac{\mathrm{T}^{\mathrm{n}+1}-\mathrm{T}^{\mathrm{n}+2 / 3}}{\Delta \mathrm{t}} \sigma \mathrm{dX}+\frac{1}{\operatorname{Re} \operatorname{Pr}} \int_{\Omega} \nabla \mathrm{T}^{\mathrm{n}+1}: \nabla \sigma \mathrm{dX}=\int_{\Omega} \mathrm{Q} \cdot \sigma \mathrm{dX}$

$$
\int_{\Omega} \frac{\mathrm{C}^{\mathrm{n}+2 / 3-\mathrm{C}^{\mathrm{n}}}}{\Delta \mathrm{t}} \sigma \mathrm{dX}+\int_{\Omega}\left(\mathrm{u}^{\mathrm{n}+1 / 3} \cdot \nabla\right) \mathrm{C}^{\mathrm{n}+2 / 3} \cdot \sigma \mathrm{dX}=0
$$

$$
\int_{\Omega} \frac{\mathrm{C}^{\mathrm{n}+1}-\mathrm{C}^{\mathrm{n}+2 / 3}}{\Delta \mathrm{t}} \sigma \mathrm{dX}+\frac{1}{\operatorname{ReSc}} \int_{\Omega} \nabla \mathrm{C}^{\mathrm{n}+1}: \nabla \sigma \mathrm{dX}=\int_{\Omega} \mathrm{R}_{\mathrm{mt}} \cdot \sigma \mathrm{dX}
$$

$\int_{\Omega} \frac{\mathrm{u}^{\mathrm{n}+1}-\mathrm{u}^{\mathrm{n}+2 / 3}}{\Delta \mathrm{t}} \cdot \sigma \mathrm{dX}+\frac{1}{\operatorname{Re}} \int_{\Omega} \nabla \mathrm{u}^{\mathrm{n}+1}: \nabla \sigma \mathrm{dX}=\operatorname{Ri} \int_{\Omega} T^{\mathrm{n}+1} \widehat{J} \cdot \sigma d X$
The system of equations above was used to obtain numerical simulations, and it stopped when the steady state was reached.

\section{ANALYSIS OF THE MESH CONVERGENCE}

The mesh dependence test for the thermal decomposition model was performed using different grids to guarantee grid-independent solutions. Mesh sizes of 21316, 24336, 27556, 30976, and 34596 nodes were used to carry out the grid sensitivity study. When comparing the solutions for grids with 21316, 24336, and 27556 nodes, considerable differences were observed; however, the analysis of the solutions for grids of 30976 and 34596 nodes indicated a variation of less than $1.2 \%$. Grid independence was verified by calculating the Nusselt number, total entropy generation, and Bejan number for the five different mesh sizes. Table 1 shows the mesh convergence analysis for two cases; in view of the results, the 30976node mesh was adopted for the ensuing calculations.

\section{RESULTS AND DISCUSSION}

Numerical simulations were conducted to obtain detailed information about the flow field inside a cavity with a chemical reaction. The main emphasis was given to the influence of Reynolds number, Schmidt number, and length of the heat source; consequently, a parametric study within the range of $\mathrm{Re}=100$ to $1000, \mathrm{Sc}=1$ to 100 , and $\mathrm{Wp}=0.2$ to 0.4 was carried out. The inside flow of the cavity was affected by three factors: fluid inlet velocity, molecular diffusivity, and heat source length. Mass transport due to mass, energy, and momentum are presented as an analysis of streamlines, isotherms, and concentration contours. The following sections present a detailed discussion of heat transfer rate in terms of Nusselt number, as well as irreversibility in terms of total entropy generation and Bejan number.

\section{Validation of Results}

\begin{tabular}{|c|c|c|c|c|c|}
\hline Nodes of the mesh & 21316 & 24336 & 27556 & 30976 & 34596 \\
\hline \multicolumn{6}{|c|}{$\mathrm{Re}=500, \mathrm{Sc}=1, \mathrm{Wp}=0.2$} \\
\hline $\mathrm{Nu}$ & 10.64533 & 11.20954 & 11.32500 & 11.39069 & 11.41085 \\
\hline$S$ & 9.51720 & 10.09775 & 10.19772 & 10.26299 & 10.28638 \\
\hline $\mathrm{Be}$ & 0.63583 & 0.67271 & 0.68011 & 0.68392 & 0.68546 \\
\hline \multicolumn{6}{|c|}{$\mathrm{Re}=1000, \mathrm{Sc}=1000, \mathrm{Wp}=0.4$} \\
\hline$N u$ & 12.39453 & 13.00187 & 13.14490 & 13.22983 & 13.25289 \\
\hline$S$ & 6.30973 & 6.67570 & 6.75581 & 6.79702 & 6.80994 \\
\hline$B e$ & 0.15373 & 0.16211 & 0.16397 & 0.16495 & 0.16523 \\
\hline
\end{tabular}

In order to validate the numerical code, two different comparisons of entropy generation and heat transfer rate were performed. The verification of the average Nusselt number has been carried out for convection

Table 1. Grid sensitivity analysis. 
heat transfer in a open cavity studied by Raji and Hasnaoui (1998). In addition, the computational code has been validated for convection in an open cavity analyzed by Manca et al. (2003). Table 2 shows good agreement when Nusselt numbers are compared. The validation of the entropy analysis has been carried out for laminar natural convection in a cavity studied by Magerbi et al. (2003). Table 3 shows good agreement when Bejan number and entropy production are compared.

\section{Fluid Dynamics}

Flow inside an open cavity, for a Schmidt number of 1.0, with a heat source in the form of a rectangular block at the bottom wall is illustrated in Figure 2. Three different heater lengths were analyzed in order to study the effect of the length of the heat source on the thermal decomposition of a substance. The left and right panels in Figure 2 show the streamlines for $\mathrm{Wp}=0.2$ and 0.4 , respectively. The top, middle, and bottom panels in Figure 2 outline the streamlines for $\mathrm{Re}=100,500$, and 1000 , respectively. Three zones of vortex formation can be identified. The first zone is behind the heater, where the inlet fluid experiments an abrupt change of the cross-sectional area, generating a clockwise vortex. The second zone is in front of the heater, where a weak clockwise vortex tends to form. The third vortex formation zone is located at the top right corner of the cavity, where an anticlockwise vortex emerges. Finally, a predominant stream moves from the inlet to the outlet of the cavity; its size changes in the central part of the cavity as a function of the Reynolds number. As expected, vortex formation intensifies and becomes more complex as the Reynolds number is increased;
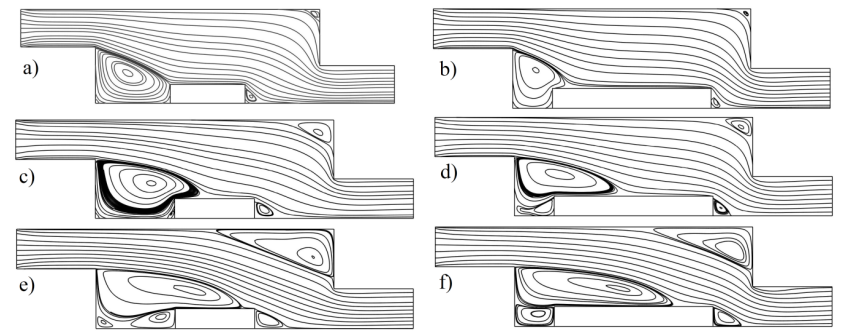

Figure 2. Streamlines for $\mathrm{Sc}=1$. a) $\mathrm{Wp}=0.2$ and $\mathrm{Re}=100$. b) $\mathrm{Wp}=0.4$ and $\mathrm{Re}=100$. c) $\mathrm{Wp}=0.2$ and $\mathrm{Re}=500$. d) $\mathrm{Wp}=0.4$ and $\mathrm{Re}=500$. e) $\mathrm{Wp}=0.2$ and $\mathrm{Re}=1000$. f) $\mathrm{Wp}=0.4$ and $\mathrm{Re}=1000$.

moreover, changing the length of the heater produces changes in the number and size of the vortices, and this behavior is most noticeable at higher Reynolds numbers. At $\mathrm{Re}=100$, only one vortex was observed in each of the three zones described above, for both $\mathrm{Wp}=0.2$ and $\mathrm{Wp}=0.4$. At $\mathrm{Re}=500$, the sizes of the vortices increase, and for $\mathrm{Wp}=0.4$, two vortices appear behind the heater. At $\mathrm{Re}=1000$, three vortices appear behind the heater for $\mathrm{Wp}=0.2$; increasing the length of the heater to $\mathrm{Wp}=0.4$ causes two vortices to appear behind the heater, of increased sizes in comparison with $\mathrm{Re}=500$. Furthermore, the size of the vortices located in front of the heater and those located at the top right corner of the cavity increase in size when the Re number is increased.

In order to provide a comprehensive insight into the dynamics of the flow, Figure 3 shows the axial velocity profiles at the middle vertical line. Figure 3a illustrates the axial velocity profile for $\mathrm{Sc}=1$ and $\mathrm{Wp}=0.2$ at different Reynolds numbers; a general parabolic behavior can be observed: the higher the Reynolds

Table 2. Validation of the numerical code based on Nusselt numbers.

\begin{tabular}{llcc}
\hline & Present & Manca et al. (2003) & Deviation \\
\hline \multirow{2}{*}{$\mathrm{Re}=100, \mathrm{Ri}=0.1$} & 1.502 (assisting flow) & 1.498 & $0.27 \%$ \\
& 1.780 (opposing flow) & 1.784 & $0.22 \%$ \\
\hline & 1.634 (heating from below) & 1.648 & $0.85 \%$ \\
\hline \multirow{2}{*}{$\mathrm{Re}=200, \mathrm{Ri}=3.472$} & Present & Raji and Hasnaoui (1998) & $0.15 \%$ \\
& 1.339 (exit by the bottom) & 1.341 & $0.77 \%$ \\
$\mathrm{Re}=50, \mathrm{Ri}=5.555$ & 1.300 (exit by the top) & 1.290 & $0.25 \%$ \\
& 1.201 (exit by the bottom) & 1.198 & $0.33 \%$ \\
\hline
\end{tabular}

Table 3. Validation of the numerical code based on entropy generation and Bejan number.

\begin{tabular}{ccccc}
\hline$\phi$ & Ra & Present & Magherbi et al. (2003) & Deviation \\
\hline & & $\mathrm{S}$ & & \\
$10^{-2}$ & $10^{3}$ & 4.524 & 4.557 & $0.72 \%$ \\
$10^{-1}$ & $10^{3}$ & 35.125 & 35.329 & $0.58 \%$ \\
$10^{-4}$ & $10^{5}$ & 22.084 & 22.223 & $0.63 \%$ \\
$10^{-3}$ & $10^{5}$ & 197.019 & 197.453 & $0.22 \%$ \\
\hline & & $\mathrm{Be}$ & \\
$10^{-2}$ & $10^{4}$ & 0.0268 & 0.0270 & $0.74 \%$ \\
$10^{-3}$ & $10^{4}$ & 0.1723 & 0.1730 & $0.40 \%$ \\
$10^{-4}$ & $10^{4}$ & 0.6767 & 0.6800 & $0.18 \%$ \\
\hline
\end{tabular}



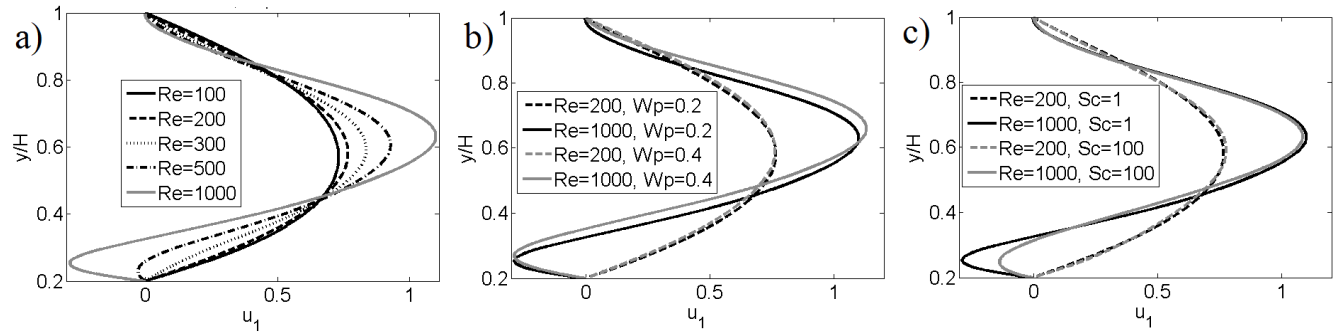

Figure 3. Axial velocity profiles at the middle vertical line as a function of the vertical coordinate. a) $\mathrm{Sc}=1, \mathrm{Wp}=0.2$. b) $\mathrm{Sc}=1$. c) $\mathrm{Wp}=0.2$.

number, the higher the maximum axial velocity value. At the highest Reynolds number, negative axial velocities are observed near the heater as a result of fluid recirculation $(\mathrm{Re}=1000)$. In this case, one of the vortices formed behind the heater is extended over the surface of the heat source, reaching the middle vertical line. Figure $3 \mathrm{~b}$ outlines the axial velocity profile for $\mathrm{Sc}=1$ with two different Reynolds numbers and two different heater lengths. At low Reynolds numbers, the effect of the length of the heater is negligible; however, at high Reynolds numbers, the length of the heater generates variations in the velocity field. At $\mathrm{Re}=1000$, axial velocity is increased toward the left, at the recirculation cell, and then, due to the predominant stream, axial velocity is reduced and the fluid changes direction; finally, axial velocity increases toward the right reaching its maximum value when $\mathrm{Wp}=0.4$. Near the top wall, axial velocity is higher for $\mathrm{Wp}=0.4$ than for $\mathrm{Wp}=0.2$. Figure $3 \mathrm{c}$ shows the axial velocity profile for $\mathrm{Wp}=0.2$ with two different Reynolds numbers and two different Schmidt numbers. No axial velocities variations are observed at low Reynolds numbers with either Schmidt number, but for $\mathrm{Re}=1000$, some variations in axial velocities are observed near the heater due to the effect of the Schmidt number variation.

\section{Thermal Characterization}

The thermal behavior over the cavity domain due to the heat released by the chemical reaction can be suitably described by the temperature fields shown in Figure 4. The left panel shows the isotherms for $\mathrm{Wp}=0.2$, while the right panel outlines the isotherms for $\mathrm{Wp}=0.4$; both panels correspond to $\mathrm{Sc}=1$. The top, middle, and bottom panels in Figure 4 outline the isotherms for $\mathrm{Re}=100,500$, and 1000 , respectively. As the Reynolds number is increased, the temperature contours tend to intensify at two zones. The first zone is associated with the heater and goes from the right end of the inlet step of the cavity to the right end of the heater. The second zone is associated with the right corner of the cavity and goes from the center of the top wall to the bottom of the right wall of the cavity. The hot temperature regions are confined to these two zones, and when the Reynolds number is increased, the

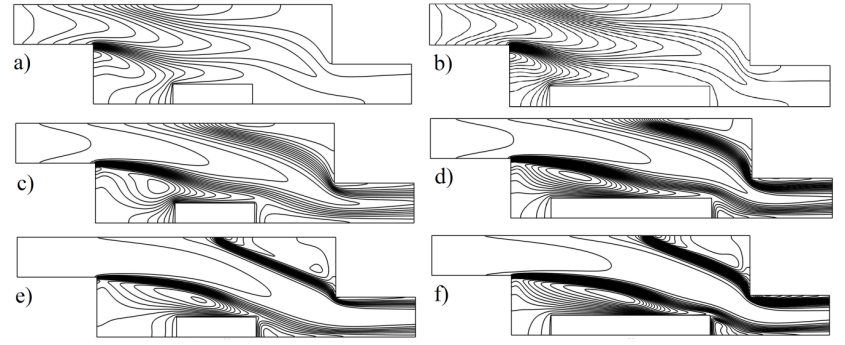

Figure 4. Isotherms for $\mathrm{Sc}=1$. a) $\mathrm{Wp}=0.2$ and $\mathrm{Re}=100$. b) $\mathrm{Wp}=0.4$ and $\mathrm{Re}=100$. c) $\mathrm{Wp}=0.2$ and $\mathrm{Re}=500$. d) $\mathrm{Wp}=0.4$ and $\mathrm{Re}=500$. e) $\mathrm{Wp}=0.2$ and $\mathrm{Re}=1000$. f) $\mathrm{Wp}=0.4$ and $\mathrm{Re}=1000$.

first one tends to move and elongate above the heater, while the second one tends to move and grow at the right corner of the cavity. The hot spot associated with the heater is driven by the chemical reaction and the energy released from the heat source, and is transported due to fluid recirculation caused by the vortices formed at the bottom left corner of the cavity. On the other hand, the hot spot associated with the right corner of the cavity depends only on the chemical reaction, and it is less intense than the other hot spot. Additionally, the temperature field is characterized by a predominant cold stream that crosses the cavity from the inlet to the outlet. This cold region increases as the inlet velocity of the fluid is augmented. Large temperature gradients occur at the interface of the hot and cold zones, regions where the contours are clustered. The isotherms also intensify when the length of the heater is increased, due to the injection of additional energy.

Flow thermal behavior can be examined in detail using Figure 5, which shows the temperature profiles at the middle vertical line. Figure 5a illustrates the temperature profile for $\mathrm{Sc}=1$ and $\mathrm{Wp}=0.2$ with different Reynolds numbers. In general, a hotter uniform temperature is observed with the lowest Reynolds number, and temperature variations strengthen as the Reynolds number is increased. Near the heater, temperature increases when the Reynolds number is increased as a result of the energy transport driven by the vortex formed above the heat source, where it reaches maximum value. Afterward, temperature starts to decrease, reaching a minimum value due to the cold temperature of the main stream. Finally, 

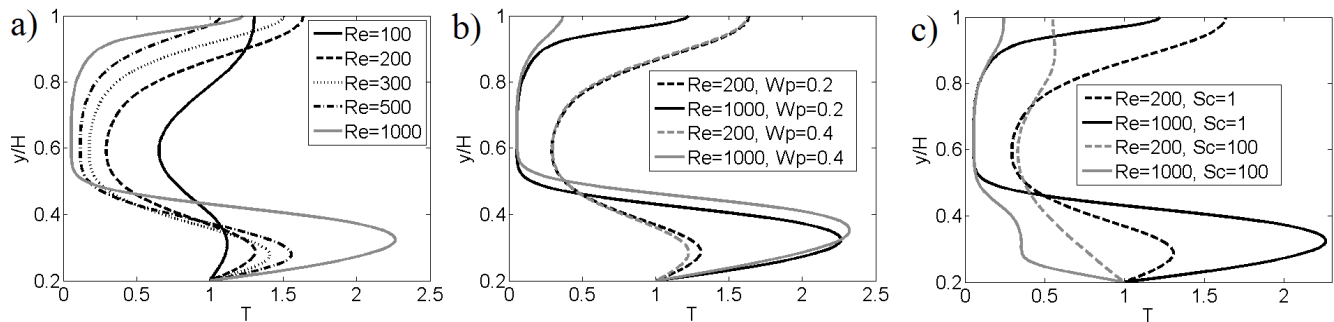

Figure 5. Temperature profiles at the middle vertical line as a function of the vertical coordinate. a) $\mathrm{Sc}=1, \mathrm{Wp}=0.2$. b) $\mathrm{Sc}=1$. c) $\mathrm{Wp}=0.2$.

temperature increases again in the neighborhood of the top wall. Figure $5 \mathrm{~b}$ outlines the temperature profile for $\mathrm{Sc}=1$ with two different Reynolds numbers and two different heater lengths. At low Reynolds numbers, small variations are observed near the heater surface, although the effects of the heater length are evident at high Reynolds numbers, and the maximum temperature is reached with the longest heater length. Figure $5 \mathrm{c}$ shows the temperature profile for $\mathrm{Wp}=0.2$ with two different Reynolds numbers and two different Schmidt numbers. At $\mathrm{Re}=200$, important variations in the temperature profiles are observed when the Schmidt number is increased from 1 to 100 . For $\mathrm{Sc}=1$, temperature increases near the heater, reaching its maximum value when $\mathrm{Re}=1000$; however, when $\mathrm{Re}=200$, temperature reaches a relative maximum value. Afterward, temperature starts to decrease to a minimum value as the vertical coordinate increases and, near the top wall, temperature goes up again, reaching its maximum value when $\mathrm{Re}=200$; however, when $\mathrm{Re}=1000$, temperature reaches a relative maximum value. At the highest Schmidt numbers, the maximum temperature value occurs on the heater surface and, afterwards, the fluid cools down to a minimum value and then it undergoes a slight increase in its temperature. At $\mathrm{Re}=1000$ and the lowest Schmidt number, the temperature profile is similar to the profile at $\mathrm{Re}=200$, although its maximum and minimum temperature values are accentuated. At $\mathrm{Re}=1000$ and the highest Schmidt number, a more complex behavior is observed near the heater than with the lowest Schmidt number.

\section{Composition Characterization}

The thermal decomposition of the substance over the cavity domain due to the chemical reaction as the flow moves through the cavity can be characterized by the concentration fields reported in Figure 6. In our model, high temperature entails low concentration, because the substance depletion is driven by temperature. The left panel in Figure 6 outlines the concentrations contours for $\mathrm{Wp}=0.2$, while the right panel shows the concentration contours for $\mathrm{Wp}=0.4$; both panels correspond to $\mathrm{Sc}=1$. The top, middle, and bottom panels in Figure 6 illustrate the concentration

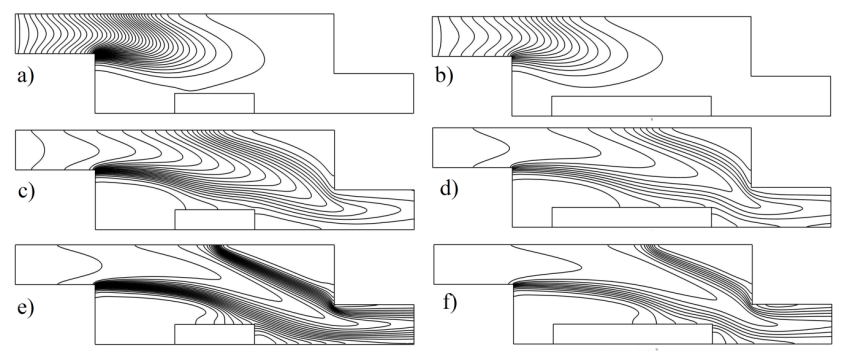

Figure 6. Concentration contours for $\mathrm{Sc}=1$. a) $\mathrm{Wp}=0.2$ and $\mathrm{Re}=100$. b) $\mathrm{Wp}=0.4$ and $\mathrm{Re}=100$. c) $\mathrm{Wp}=0.2$ and $\mathrm{Re}=500$. d) $\mathrm{Wp}=0.4$ and $\mathrm{Re}=500$. e) $\mathrm{Wp}=0.2$ and $\mathrm{Re}=1000$. f) $\mathrm{Wp}=0.4$ and $\mathrm{Re}=1000$.

contours for $\mathrm{Re}=100,500$, and 1000 , respectively. The concentration contours decline as the length of the heater is increased, since the amount of energy injected into the fluid is larger than when the heater is smaller. The longer the heater, the greater the decomposition. Furthermore, it can be observed that concentration contours weaken at lower Reynolds numbers, that is, the decomposition of the substance is increased when the velocity of the fluid is small because the center of the cavity is heated uniformly. At higher Reynolds numbers, a cold stream predominates, preventing thermal decomposition from taking place at the center of the cavity. Moreover, large concentration gradients develop at the hot/cold flow interface.

To better illustrate substance decomposition, Figure 7 shows the concentration profiles at the middle vertical line. Figure 7 a shows the concentration profile for $\mathrm{Sc}=1$ and $\mathrm{Wp}=0.2$ with different Reynolds numbers; substance concentration is almost zero at lower Reynolds numbers, which means that thermal decomposition is highest. Upon increasing the Reynolds number, the substance decomposition is reduced, principally at the center of the predominant cold stream; however, substance depletion is high near the heater. At the top wall of the cavity, thermal decomposition increases gradually as the Reynolds number decreases. Figure $7 \mathrm{~b}$ outlines the temperature profile for $\mathrm{Sc}=1$ with two different Reynolds numbers and two different heater lengths. At low Reynolds numbers, concentration profiles do not show differences when the length of the heater is changed; however, important variations are observed 

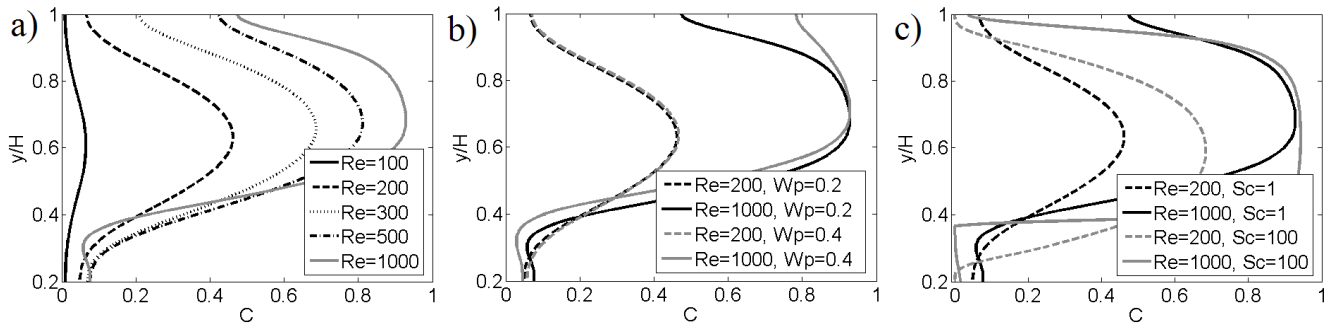

Figure 7. Concentration profiles at the middle vertical line as a function of the vertical coordinate. a) $\mathrm{Sc}=1, \mathrm{Wp}=0.2$. b) $\mathrm{Sc}=1$. c) $\mathrm{Wp}=0.2$.

at high Reynolds numbers due to the effect of the heat source length. At $\mathrm{Re}=1000$, concentration is higher for $\mathrm{Wp}=0.2$ than for $\mathrm{Wp}=0.4$ from the heater surface to the center of the middle vertical line, and beyond that point, concentration is higher for $\mathrm{Wp}=0.4$ than for $\mathrm{Wp}=0.2$. Figure $7 \mathrm{c}$ shows the concentration profile for $\mathrm{Wp}=0.2$ with two different Reynolds numbers and two different Schmidt numbers. For $\mathrm{Re}=200$, concentration increases when the Schmidt number is increased in almost all the middle vertical line, which indicates that thermal decomposition (low concentration values) increases with low Schmidt numbers. More complex profiles are observed at $\mathrm{Re}=1000$; near the heater, in a small zone, the concentration of the substance is lower at $\mathrm{Sc}=100$ than at $\mathrm{Sc}=1$, and beyond $\mathrm{y} / \mathrm{H}=0.4$, substance depletion (thermal decomposition) is higher at $\mathrm{Sc}=1$ than at $\mathrm{Sc}=100$. In the neighborhood of the top wall, again in a small region, the concentration of the substance is reduced with high Schmidt numbers. It can also be observed that, at $\mathrm{Sc}=100$, in a large region from $\mathrm{y} / \mathrm{H}=0.4$ to 0.9 , concentration values are high, in fact reaching maximum value. Therefore, thermal decomposition, which entails low concentration values, is enhanced at low Schmidt numbers.

\section{Heat Transfer Rate Analysis}

Figure 8 outlines heat transfer characteristics in the cavity where flow is subjected to thermal decomposition, through the variation in the average Nusselt number as a function of the Reynolds number for different heater lengths, and several Schmidt numbers. Figures $8 \mathrm{a}$ and $8 \mathrm{~b}$ show average Nusselt numbers for $\mathrm{Sc}=1$ and $\mathrm{Sc}=10$, respectively. Figures $8 \mathrm{c}$ and $8 \mathrm{~d}$ show average Nusselt numbers for $\mathrm{Sc}=100$ and $\mathrm{Sc}=1000$, respectively. It is clear from these figures that the Nusselt number increases as the Reynolds number grows for all heater lengths and all Schmidt numbers studied. When keeping Reynolds number constant, the heat transfer rate is increased as the heater length increases due to the higher energy input. At lower Reynolds numbers, the variations in $\mathrm{Nu}$ with the length of the heater are lesser than with higher Reynolds numbers. Increasing the Reynolds number makes the cooling of the cavity more effective: large zones of cold fluid are observed, axially crossing the
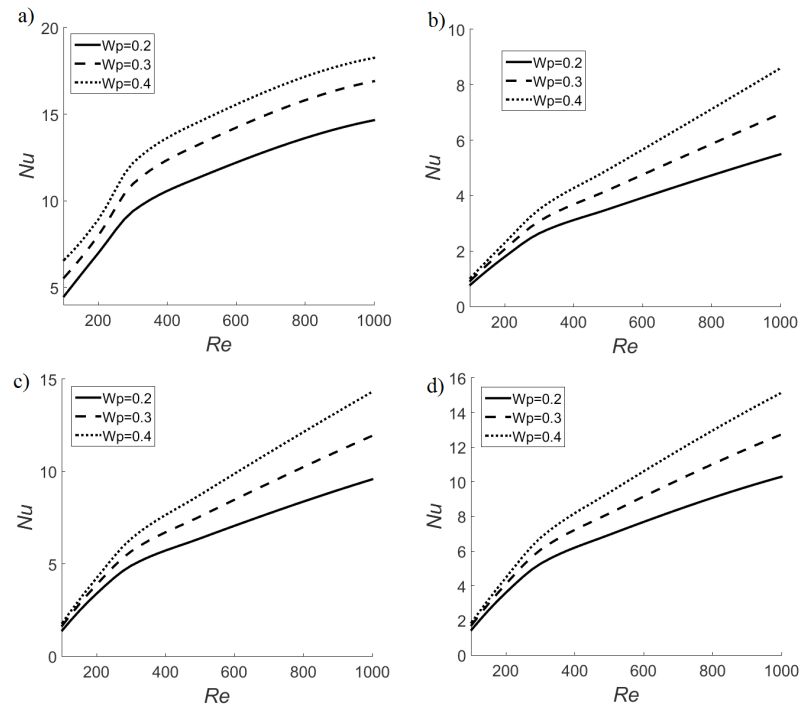

Figure 8. Average Nusselt number as a function of the Reynolds number for different lengths of the heater. a) $\mathrm{Sc}=1$. b) $\mathrm{Sc}=10$. c) $\mathrm{Sc}=50$. d) $\mathrm{Sc}=100$.

cavity and preventing thermal decomposition from taking place inside the cavity. In other words, the concentration of the substance is high when Nusselt numbers are high due to a more effective fluid cooling. The released energy also increases as the heater is elongated, given that vortices increase in number and motion, thus improving energy transport.

The variation of the average Nusselt number as a function of the Schmidt number at different Reynolds numbers and different lengths of the heater is shown in Figure 9, which shows that the

heat transfer rate varies drastically when $1 \leq \mathrm{Sc}$ $\leq 10$, reaching a minimal value at $\mathrm{Sc}=10$. When $\mathrm{Sc}$ $>10$, the average Nusselt number goes up when the Schmidt number is increased to $\mathrm{Sc}=50$, and afterward, the heat transfer rate undergoes a slight increase. The above behavior implies that fluid cooling is minimum at $\mathrm{Sc}=10$; as a consequence, the temperature of the fluid inside the cavity is highest, promoting thermal decomposition. On the other hand, when comparing the average Nusselt number for the three different heater lengths, it can be observed that longer heaters result in higher heat transfer rates from the heater to the system. 

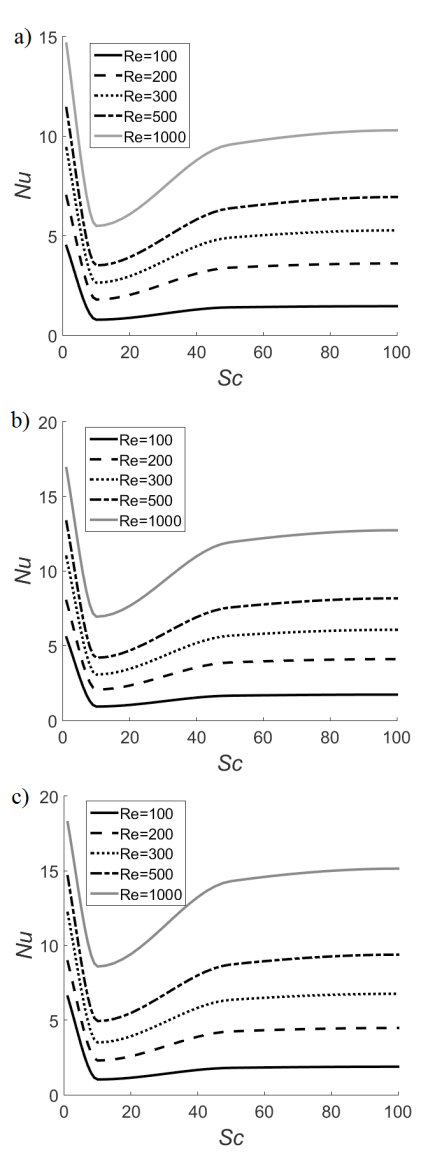

Figure 9. Average Nusselt number as a function of the Schmidt number for different Reynolds numbers. a) $\mathrm{Wp}=0.2$. b) $\mathrm{Wp}=0.3$. c) $\mathrm{Wp}=0.4$.

\section{Entropy Generation Analysis}

The irreversibility of the thermal decomposition due to a chemical reaction in flow inside the cavity was analyzed by means of total entropy generation and Bejan number maps; see left and right panels in Figure 10, respectively. Figure 10a shows total entropy generation for $\mathrm{Sc}=1, \mathrm{Wp}=0.2$, and $\mathrm{Re}=100$. Strong irreversibilities can be observed at the entrance of the cavity, at the corner where the fluid undergoes a big expansion due to the change in the crosssectional area, on the surface of the heater and at the exit of the cavity. When $\mathrm{Sc}$ and $\mathrm{Wp}$ are maintained constant and Reynolds number is 1000 , as shown in Figure 10c, the main sources of irreversibility place themselves in the upper right corner of the inlet step and the lower corner of the right wall of the cavity, and so two bands of irreversibility are identified in the interface between the predominant cold stream and the zones of vortices due to the large gradients occurring in these regions. By increasing the length of the heater to $\mathrm{Wp}=0.4$ and holding $\mathrm{Sc}=1$ and $\mathrm{Re}=100$ (see Figure 10e), irreversibility increases on the heater surface and intensifies at the entrance and exit of the cavity due to the blockage effect, i.e., when the heater increases in length, the fluid is accelerated due to a
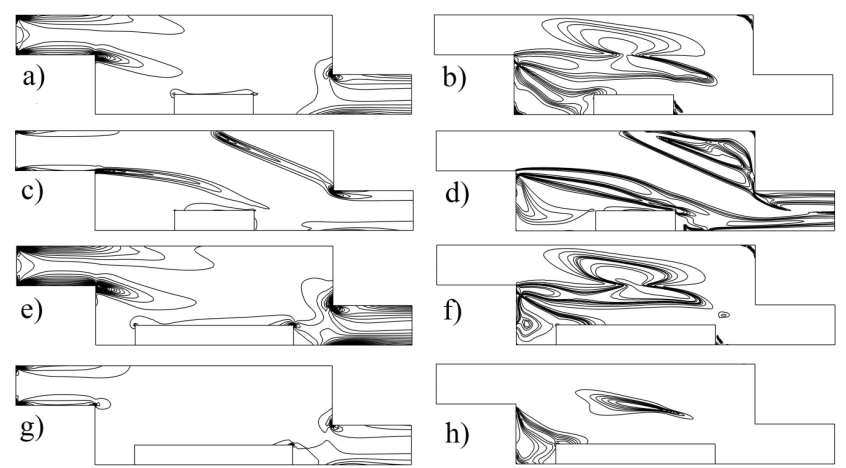

Figure 10. Total entropy generation maps (left) and Bejan number maps (right). a) and b) $\mathrm{Sc}=1, \mathrm{Wp}=0.2$, $\mathrm{Re}=100 . \mathrm{c})$ and d) $\mathrm{Sc}=1, \mathrm{Wp}=0.2, \mathrm{Re}=1000$. e) and f) $\mathrm{Sc}=1, \mathrm{Wp}=0.4, \mathrm{Re}=100 . \mathrm{g}$ ) and $\mathrm{h}) \mathrm{Sc}=100, \mathrm{Wp}=0.4$, $\mathrm{Re}=100$.

reduction of area between the upper right corner of the heater and the lower corner of the right wall of the cavity, and so the fluid undergoes more friction. When $\mathrm{Wp}=0.4$ and $\mathrm{Re}=100$ are maintained constant and the Schmidt number is increased to 100 (see Figure $10 \mathrm{~g}$ ), irreversibility inside the cavity is attenuated due to a decline in mass diffusivity.

Figure 10b shows the Bejan number for $\mathrm{Sc}=1$, $\mathrm{Wp}=0.2$, and $\mathrm{Re}=100$, and identifies the regions where heat transfer irreversibility is predominant: two zones of strong thermal irreversibility emerging from the right end of the inlet step of the cavity. The first region is near the center of the cavity and extends upward. The second region is near the left wall of the heater and extends to the bottom left corner of the cavity. Contours concentrate in both zones, which indicates large gradients of temperature in these regions of the cavity. When Sc and Wp are maintained constant and the Reynolds number is increased to 1000 (see Figure 10d), large regions of heat transfer irreversibility appear at the upper right corner of the cavity and in the region that extends from the upper corner of the left wall of the cavity to the top right corner of the heater; this thermal irreversibility is due to large gradients occurring in the interface between the predominant cold stream and the hot vortices, which are intensified by the increased Reynolds number. Increasing the length of the heater to $\mathrm{Wp}=0.4$ and holding $\mathrm{Sc}=1$ and $\mathrm{Re}=100$ (see Figure 10f) causes Bejan contours to behave as in the case of $\mathrm{Wp}=0.2$ at the same $\mathrm{Sc}$ and $\mathrm{Re}$; however, these contours are more intense, since a larger heater involves higher energy injected into the fluid than with a small heater. Maintaining $\mathrm{Wp}=0.4$ and $R e=100$ constant and increasing Schmidt number to 100 (see Figure 10h) causes a small region at the center of the cavity and a region behind the heater to exhibit the highest temperature gradients.

Average total entropy generation is shown in Figure 11 as a function of the Reynolds number for different 

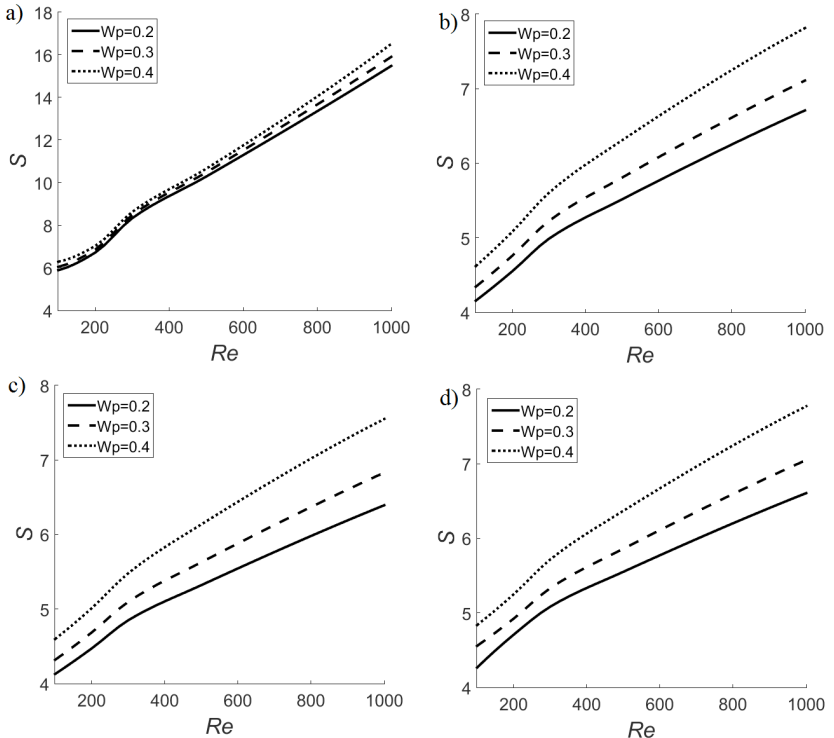

Figure 11. Global total entropy generation as a function of the Reynolds number for different lengths of the heater. a) $\mathrm{Sc}=1$. b) $\mathrm{Sc}=10$. c) $\mathrm{Sc}=50$. d) $\mathrm{Sc}=100$.

heater lengths and different Schmidt numbers. All cases indicate that average total entropy generation increases as the Reynolds number increases. This behavior is due to the fact that flow patterns become more complex as the Reynolds number increases, generating more energy transport with stronger velocity and thermal gradients. Furthermore, average total irreversibility increases as the length of the heat source increases due to the larger amount of energy injected into the system, and also, the longer the heater, the stronger the flow perturbation. The maximum values of average total entropy generation were obtained at $\mathrm{Sc}=1$ (see Figure 11a), although the variations due to changes in the length of the heater for a given Reynolds number are small. When increasing the Schmidt number to $\mathrm{Sc}=10$ (see Figure 11b), average total entropy generation is reduced in comparison with the previous case, although the variations due to changes in the length of the heater for a given Reynolds number increase. Figures $11 \mathrm{c}$ and $11 \mathrm{~d}$ show average total entropy generation for $\mathrm{Sc}=50$ and 100 , respectively. The examination of these two figures indicates that, beyond $\mathrm{Sc}=10$, average total entropy generation varies slightly for a given Reynolds number and a given length of the heater.

The variation in average total entropy generation when the Schmidt number is increased, at different Reynolds numbers and different heater lengths, is shown in Figure 12. Figure 12a is for $\mathrm{Wp}=0.2$, Figure $12 b$ is for $\mathrm{Wp}=0.3$ and Figure $12 \mathrm{c}$ is for $\mathrm{Wp}=0.4$. The largest average total entropy generation occurs at $\mathrm{Sc}=1$; beyond this value, entropy generation undergoes a rapid descent until $\mathrm{Sc}=10$, after which a slight descent is observed and reaches its minimum value when $45<\mathrm{Sc}<55$, and finally, a slight increase can be appreciated. Furthermore, the minimum value
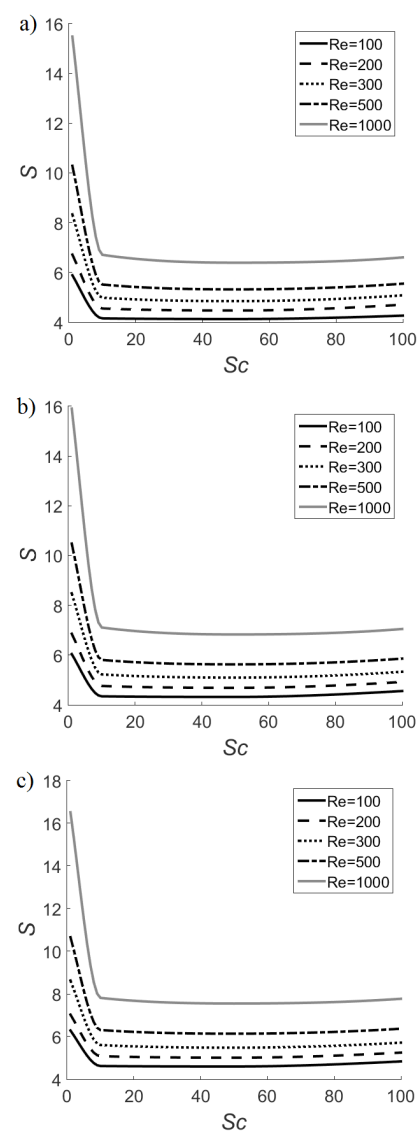

Figure 12. Global total entropy generation as a function of the Schmidt number for different Reynolds numbers. a) $\mathrm{Wp}=0.2$. b) $\mathrm{Wp}=0.3$. c) $\mathrm{Wp}=0.4$.

of the average total entropy generation, for a given length of the heater occurs at the lowest Reynolds number. Flow composition characterization showed that thermal decomposition is greater at the lowest Re, highest $\mathrm{Wp}$, and lowest $\mathrm{Sc}$; however, with regard to the entropy production analysis, irreversibilities are minimal at the lowest $\mathrm{Re}$, lowest $\mathrm{Wp}$, and $45<\mathrm{Sc}<55$. Therefore, from the point of view of energy efficiency, the optimal design of an open cavity with a chemical reaction where thermal decomposition takes place is: $45<\mathrm{Sc}<55, \mathrm{Re}=100$ and $\mathrm{Wp}=0.2$.

Figure 13 reveals the effect of fluid dynamics on heat transfer irreversibility by means of average Bejan number versus Reynolds number, with different heat source lengths and various Schmidt numbers. Figures $13 \mathrm{a}$ and $13 \mathrm{~b}$ show the average Bejan numbers for $\mathrm{Sc}=1$ and $\mathrm{Sc}=10$, respectively. Figures $13 \mathrm{c}$ and $13 \mathrm{~d}$ show the average Bejan numbers for $\mathrm{Sc}=50$ and $\mathrm{Sc}=100$, respectively. As the Reynolds number increases, the average Bejan number increases due to strong thermal gradients developed as a result of the interaction of the cold stream with the hot regions of the flow; this behavior continues until approximately $\mathrm{Re}=300$, where heat transfer irreversibility reaches its maximum value. Afterward, Be decreases slightly, 

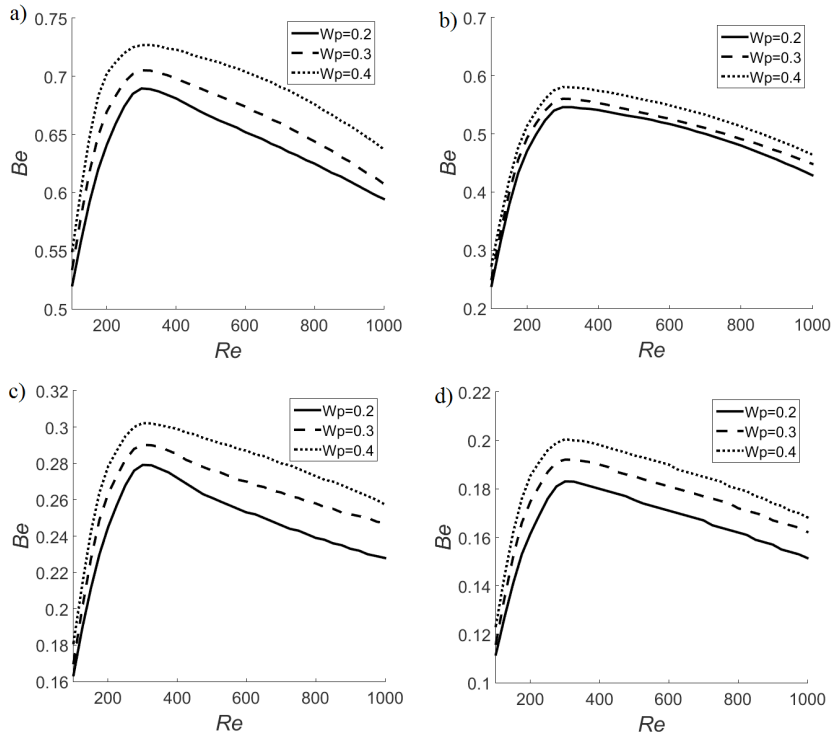

Figure 13. Average Bejan number as a function of the Reynolds number for different lengths of the heater. a) $\mathrm{Sc}=1$. b) $\mathrm{Sc}=10$. c) $\mathrm{Sc}=50$. d) $\mathrm{Sc}=100$.

whereas Re increases because the inlet cold stream covers a large area, maintaining uniform temperatures in the center of the cavity. The average Bejan number is also characterized by an increase when the length of the heater is increased, due to higher energy transport.

The effect of Schmidt number on average Bejan number at different Reynolds numbers and heater lengths is illustrated in Figure 14. Figure 14a is for $\mathrm{Wp}=0.2$, Figure $14 \mathrm{~b}$ is for $\mathrm{Wp}=0.3$ and Figure $14 \mathrm{c}$ is for $\mathrm{Wp}=0.4$. These panels indicate that, as the mass diffusion rate decreases and the Schmidt number increases, the average Bejan number declines. Thermal irreversibility decreases quickly when $\mathrm{Sc}<$ 10 , and beyond this value, a slight descent of $\mathrm{Be}$ is observed in all cases. If $\mathrm{Wp}$ is maintained constant, Bejan number variations are reduced as the Reynolds number is increased.

The three different components of the total entropy generation for $\mathrm{Sc}=1, \mathrm{Wp}=0.2$ and $\mathrm{Re}=100$ are shown in Figure 15. Irreversibilities due to friction of the fluid are illustrated in Figure 15a, noting that at the entrance and at the exit the velocity gradients are intensified, especially towards the walls due to the increase in friction in the areas where the crosssectional area of the channel is reduced. Figure $15 \mathrm{~b}$ describes the irreversibilities due to heat transfer; the contours suggest that the temperature gradients gradually intensify from the entrance of the channel and become more intense in the area between the corner of the channel step and the central part of the channel where the interaction between the heat flux of the heater and the incoming cold flow of the fluid cause high temperature gradients. Figure 15c shows the generation of entropy due to concentration
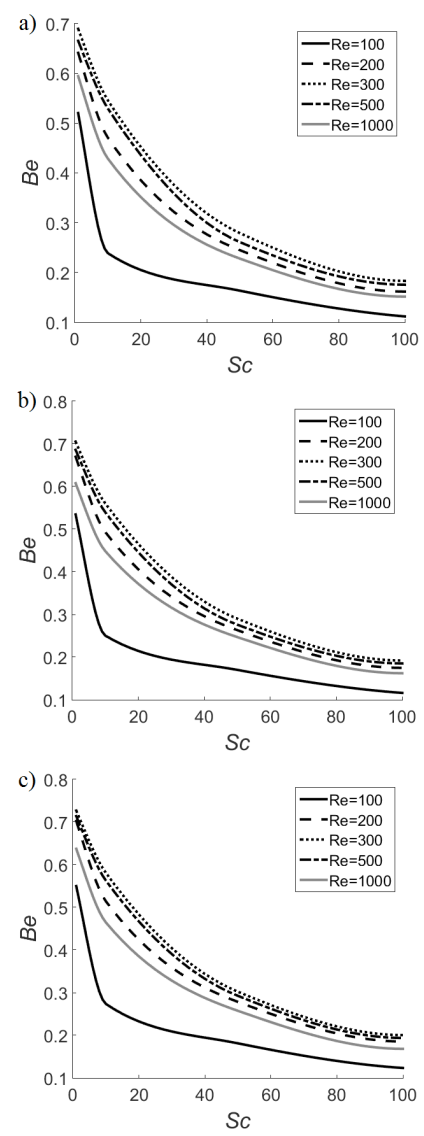

Figure 14. Average Bejan number as a function of the Schmidt number for different Reynolds numbers. a) $\mathrm{Wp}=0.2$. b) $\mathrm{Wp}=0.3$. c) $\mathrm{Wp}=0.4$.

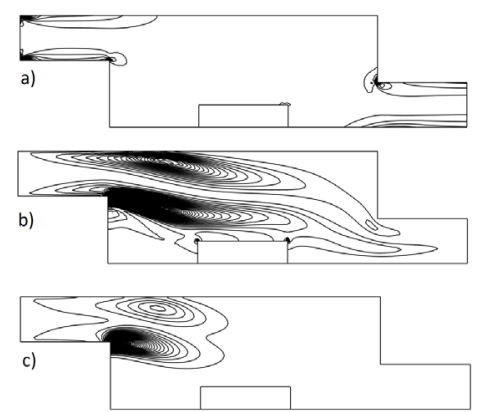

Figure 15. a) Entropy generation due to fluid flow, b) Entropy generation due to heat transfer and c) Entropy generation due to mass transfer for $\mathrm{Sc}=1, \mathrm{Wp}=0.2$, $\mathrm{Re}=100$.

gradients. These gradients are important in the initial part of the channel and to the beginning of the heater because in this area most of the thermal decomposition takes place. It is important to note that the total entropy map for this case tends to resemble the entropy map for heat transfer in the central part of the channel, observing elongated contours from the corner of the channel step that project to the heater, on which also entropy generation contours can be observed. On the other hand, at the entrance and exit of the channel, the 
total entropy generation map follows the behavior of the entropy by fluid friction.

Figure 16 shows the entropy maps due to viscous, thermal and concentration effects for $\mathrm{Sc}=100, \mathrm{Wp}=0.4$ and $\mathrm{Re}=100$. Figure 16a indicates that the friction losses are lower in the central part of the channel and increase in the entrance and exit of the channel. Figure $16 \mathrm{~b}$ shows that the irreversibilities for heat transfer are important after the step of the channel and on the heating plate. This is because the temperature of the fluid in the recirculation zone generated behind the heating plate is higher than the one of the incoming stream. Figure $16 \mathrm{c}$ indicates that the irreversibilities for concentration intensify at the beginning of the channel where the variations from mass diffusion are relevant. Later, as the fluid moves, the gradients due to the concentration are gradually reduced. For this case, the total entropy generation map is similar to the entropy generation map due to fluid friction.

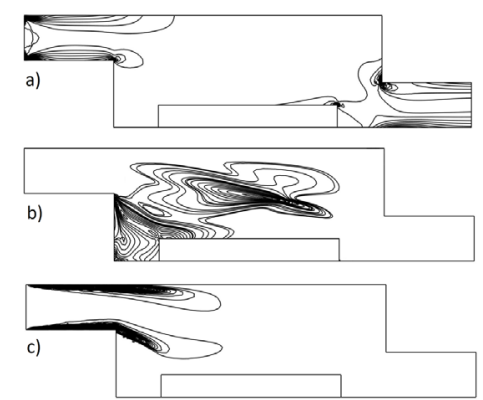

Figure 16. a) Entropy generation due to fluid flow, b) Entropy generation due to heat transfer and c) Entropy generation due to mass transfer for $\mathrm{Sc}=100, \mathrm{Wp}=0.4$, $\mathrm{Re}=100$.

\section{CONCLUSIONS}

This paper presents a two-dimensional steadystate numerical study of the entropy generated by fluid friction, heat transfer, and mass transfer in a ventilated cavity with thermal decomposition driven by a chemical reaction. The fluid enters the cavity in a state of high concentration, and as a result of the chemical reaction, its concentration decays as it moves through the cavity over a heat source placed at the bottom wall, where concentration insulation boundary conditions are imposed. Fluid dynamics, heat, mass transport, and their associated irreversibilities are significantly influenced by the Reynolds number, the Schmidt number, and the length of the heat source. The streamlines and velocity profiles show the complex pattern of the flow interacting with heat and mass transport. Temperature and concentration profiles and maps evince a strong dependency on the Re and Sc numbers, and a weak dependency on the length of the heater. As the Reynolds number increases, cooling inside the cavity becomes more effective, and large zones of cold fluid are observed axially crossing the cavity and preventing thermal decomposition from taking place inside the cavity. Variations in heat transfer rate display a more complex behavior when the Schmidt number goes from 1 to 100 , reaching a minimal value when $\mathrm{Sc}=10$. Average total entropy generation profiles indicate that irreversibilities are reduced when the Reynolds number decreases. Moreover, the shorter the heater the less the average total entropy production. From $\mathrm{Sc}=1$ to $\mathrm{Sc}=10$, a steep decrease in entropy production is observed, while from $10<\mathrm{Sc} \leq 100$, a slight variation can be appreciated, although minimal values of entropy production are reached when $45 \leq \mathrm{Sc} \leq 55$. Average Bejan number profiles show that thermal irreversibilities reach a maximum value when $\mathrm{Re}=300$. The longer the heater, the more the thermal irreversibilities caused. Furthermore, the average Bejan number decreases with the increase of the Schmidt number. The results indicate that the maximal efficiency of the thermal decomposition inside an open cavity occurs when $45<\mathrm{Sc}<55$, with the lowest Reynolds number and the smallest size of the heater. Since the research presented in this paper is limited to the geometry and the range of parameters used in the study, ongoing research includes three-dimensional simulations and multiphase flows. The foremost advantage of the present study is its possible application in minimizing costs of energy in industrial processes.

\section{ACKNOWLEDGEMENTS}

This research was supported by PRODEPCONACYT-TNM-ITVer (VER-PYR-2016-090).

\section{NOMENCLATURE}

A pre-exponential factor, $\mathrm{s}^{-1}$

$\mathrm{Be} \quad$ Bejan number, dimensionless

$\mathrm{C}_{\mathrm{B}}$ specific heat capacity, $\mathrm{J} \mathrm{kg}^{-1} \mathrm{~K}^{-1}$

$\mathrm{C}^{\mathrm{p}}$ mass concentration, $\mathrm{mol} \mathrm{m}^{-3}$

C mass concentration, dimensionless

dA differential area, dimensionless

D diffusion coefficient, $\mathrm{m}^{2} \mathrm{~s}^{-1}$

E activation energy, $\mathrm{J} \mathrm{mol}^{-1}$

g gravity, $\mathrm{m} \mathrm{s}^{-2}$

$\mathrm{H} \quad$ cavity height, $\mathrm{m}$

$\hat{\mathrm{J}} \quad$ vertical unit vector, dimensionless

k' thermal conductivity, $\mathrm{W} \mathrm{m}^{-1} \mathrm{~K}^{-1}$

$\mathrm{k}$ thermal conductivity, dimensionless

$\mathrm{n} \quad$ time step

$\mathrm{Nu} \quad$ Nusselt number, dimensionless

$\mathrm{p}$ pressure, dimensionless

$\mathrm{P}$ pressure, $\mathrm{Pa}$

Pr Prandtl number, dimensionless

Q source term of the energy equation, $\mathrm{J} \mathrm{m}^{-3} \mathrm{~s}^{-1}$ 
$r \quad$ reaction rate, $\mathrm{mol} \mathrm{m}^{-3} \mathrm{~s}^{-1}$

$\mathrm{R}_{\mathrm{mt}} \quad$ source term of the mass transport equation, mol m-3 $\mathrm{s}^{-1}$

$\mathrm{R}_{\mathrm{g}} \quad$ universal gas constant, $\mathrm{J} \mathrm{mol}^{-1} \mathrm{~K}^{-1}$

$\mathrm{Re} \quad$ Reynolds number, dimensionless

$\mathrm{Ri} \quad$ Richardson number, dimensionless

$\mathrm{S} \quad$ entropy generation, dimensionless

T' temperature, $\mathrm{K}$

$\mathrm{T}$ temperature, dimensionless

$\mathrm{t}$ time, dimensionless

t time, $\mathrm{s}$

$\mathrm{U}_{\text {in }} \quad$ inlet flow velocity, $\mathrm{m} \mathrm{s}^{-1}$

$\mathrm{u}$ velocity vector, dimensionless

$\mathrm{u}_{1} \quad$ horizontal velocity component, dimensionless

$\mathrm{u}_{1}$ horizontal velocity component, $\mathrm{m} \mathrm{s}^{-1}$

$\mathrm{u}_{2} \quad$ vertical velocity component, dimensionless

$\mathrm{u}_{2} \quad$ vertical velocity component, $\mathrm{m} \mathrm{s}^{-1}$

W length of the cavity, $\mathrm{m}$

$\mathrm{W}_{\mathrm{p}}$ length of the heater, dimensionless

$\mathrm{X}^{\mathrm{p}}$ position vector, dimensionless

$\mathrm{x}$ horizontal coordinate, dimensionless

$\mathrm{x}$ ' horizontal coordinate, $\mathrm{m}$

y vertical coordinate, dimensionless

y' vertical coordinate, $\mathrm{m}$

\section{Greek symbols}

$\beta \quad$ compressibility coefficient, $\mathrm{K}^{-1}$

$v \quad$ kinematic viscosity, $\mathrm{m}^{2} \mathrm{~s}^{-1}$

$\gamma \quad$ rate constant. $\mathrm{s}^{-1}$

$\rho$ density, $\mathrm{kg} \mathrm{m}^{-3}$

$\sigma \quad$ weighting function of the finite element, dimensionless

$\varphi \quad$ irreversibility ratio, dimensionless

$\Phi \quad$ heat of reaction, $\mathrm{J} \mathrm{mol}^{-1}$

$\Omega \quad$ two-dimensional domain, dimensionless

$\mu$ dynamic viscosity, $\mathrm{Pa} \mathrm{s}$

$\mu \quad$ dynamic viscosity, dimensionless

\section{Subscripts}

c cool

ff fluid friction

h hot

$\mathrm{H}$ high

ht heat transfer

1 local

$\mathrm{mt}$ mass transfer

ref reference

htl local entropy generation due to heat transfer

ffl local entropy generation due to fluid flow

$\mathrm{mtl}$ local entropy generation due to mass transfer

\section{REFERENCES}

Anderson, J. D., Computational fluid dynamics, McGraw-Hill, USA (1995).
Bermejo, P., Revellin, R., Charnay, R., Garbrecht, O., Hugon, J., Bonjour, J., Modeling of a Microchannel Evaporator for Space Electronics Cooling: Entropy Generation Minimization Approach, Heat Transfer Engineering 34(4), 303-312 (2013). https://doi.org/ 10.1080/01457632.2012.715069

Bousri, A., Nebbali, R., Bennacer, R., Bouhadef, K., Beji, H., Numerical Investigation of Forced Convection Nonequilibrium Effects on Heat and Mass Transfer in Porous Media, Heat Transfer Engineering, 38(1), 122-136 (2017). https://doi.or $\mathrm{g} / 10.1080 / 01457632.2016 .1156422$

Cheddie, D., Ammonia as a Hydrogen Source for Fuel Cells: a Review, Chapter 13 in Hydrogen EnergyChallenges and Perspectives, Ed. Minic, D., 333362 (2012). https://doi.org/10.5772/47759

Chen, C. K., Chen, B. S., Liu, C. C., Heat Transfer and Entropy Generation in Fully-Developed Mixed Convection Nanofluid Flow in Vertical Channel, International Journal of Heat and Mass Transfer, 79, 750-758 (2014). https://doi.org/10.1016/j. ijheatmasstransfer.2014.08.078

Chen, S., Yang, B., Xiao, X., Zheng, C., Analysis of Entropy Generation in Double-Diffusive Natural Convection of Nanofluid, International Journal of Heat and Mass Transfer, 87, 447-463 (2015). https:// doi.org/10.1016/j.ijheatmasstransfer.2015.04.023

Cheng, C. H., Huang, W. H., Entropy Generation and Heat Transfers Via Laminar Forced-Convection Channel Flows over Transverse Fins in Entrance Regions, Applied Energy, 32(4), 241-267 (1989). https://doi.org/10.1016/0306-2619(89)90015-9

Dhiman, A. K., Sharma, N., Kumar, S., Wall Effects on the Ccross-Buoyancy around a Square Cylinder in the Steady Regime, Brazilian Journal of Chemical Engineering, 29(2), 253-264 (2012). https://doi. org/10.1590/S0104-66322012000200006

Duan, H. Y., Hsieh, P. W., Tan, R. C. E., Yang, S. Y., Analysis of the Small Viscosity and Large Reaction Coefficient in the Computation of the Generalized Stokes Problem by a Novel Stabilized Finite Element Method, Computer Methods in Applied Mechanics and Engineering, 271, 23-47 (2014). https://doi.org/10.1016/j.cma.2013.11.024

Fontana, E., Mancusi, E., Ulson de Souza, A. A., Guelli U. Sousa, S. M. A., Linear Stability Analysis and CFD Simulation of Double-Layer RayleighBenard Convection, Brazilian Journal of Chemical Engineering, 33(3), 607-616 (2016). https://doi. org/10.1590/0104-6632.20160333s20150050

Ganesan, S., An Operator-Splitting Galerkin/SUPG Finite Elemnt Method for Population Balance Equations: Stability and Convergence, ESAIMMathematical Modelling and Numerical Analysis, 46, 1447-1465 (2012). https://doi.org/10.1051/ $\mathrm{m} 2 \mathrm{an} / 2012012$ 
Ganesan, S., Tobiska, L., An Operator-Splitting Finite Element Method for the Efficient Parallel Solution of Multidimensional Population Balance Systems, Chemical Engineering Science, 69, 59-68 (2012). https://doi.org/10.1016/j.ces.2011.09.031

Ghachem, K., Kolsi, L., Maatki, C., Hussein, A. K., Borjini, M. N., Numerical Simulation of ThreeDimensional Double Diffusive Free Convection flow and Irreversibility studies in a Solar Distiller, International Communications in Heat and Mass Transfer, 39, 869-876 (2012). https://doi. org/10.1016/j.icheatmasstransfer.2012.04.010

Groor, S. R., Mazur, P., Non-Equilibrium Thermodynamics, Dover, New York (1984).

Harfash, A. J., Alshara, A. K., Chemical Reaction Effect on Double Diffusive Convection in Porous Media with Magnetic and Variable Gravity Effects, Korean Journal. Chemical. Engineering, 32(6), 1046-1059 (2015). https://doi.org/10.1007/s11814014-0327-5

Hauke, G., Sangalli, G., Doweidar, M. H., Combining Adjoint Stabilized Methods for the AdvectionDiffusion-Reaction Problem, Mathematical Models and Methods in Applied Sciences, 17, 305-326 (2007). https://doi.org/10.1142/ S0218202507001929

Khan, W. A., Gorla, R. S. R., Second Law Analysis for Free Convection in Non-newtonian Fluid over a Horizontal Plate Embedded in a Porous Medium: (Prescribed Heat Flux). Brazilian Journal of Chemical Engineering, 29(3), 511$518 \quad$ (2012). https://doi.org/10.1590/S010466322012000300008

Lazarovici, A., Volpert, V., Merkin, J. H., Steady States, Oscillations and Heat Explosion in a Combustion Problem with Convection, European Journal of Mechanics B-Fluids, 24, 189-203 (2005). https:// doi.org/10.1016/j.euromechflu.2004.06.007

Lin, C. H., Cheng, W. T., Lee, J. H., Effect of Embedding a Porous Medium on the Deposition Rate in a Vertical Rotating MOCVD Reactor Based on CFD Modeling, International Communication in Heat and Mass Transfer, 36(7), 680-685 (2009). https:// doi.org/10.1016/j.icheatmasstransfer.2009.03.019

Luo, G., Vanka, S. P., Glumac, N., Fluid Flow and Transport Processes in a Large Area Atmospheric Pressure Stagnation Flow CVD Reactor for Deposition of Thin Films, International Journal of Heat and Mass Transfer, 47(23), 4979-4994 (2004). https://doi.org/10.1016/j. ijheatmasstransfer.2004.06.012

Magherbi, M., Abbassi, H., Ben Brahim, A., Entropy Generation at the Onset of Natural Convection, International Journal of Heat and Mass Transfer, 46(18), 3441-3450 (2003). https://doi.org/10.1016/ S0017-9310(03)00133-9
Mahmud, S., Fraser, R.A., Flow, Thermal and Entropy Generation Characteristics inside a Porous Channel with Viscous Dissipation, International Journal of Thermal Sciences, 44, 21-32 (2005). https://doi. org/10.1016/j.ijthermalsci.2004.05.001

Manca, O., Nardini, S., Khanafer, K., Vafai, K., Effect of Heated Wall Position on Mixed Convection in a Channel with an Open Cavity, Numerical Heat Transfer, Part A: Applications, 43, 259-282, (2003). https://doi.org/10.1080/10407780307310

Mariani, V.C., Coelho, L. S., Natural Convection Heat Transfer in Partially Open Enclosures Containing an Internal Local Heat Source, Brazilian Journal of Chemical Engineering, 24(3), 375-388 (2007). https://doi.org/10.1590/S010466322007000300007

Rahman, M. M., Parvin S., Hasanuzzaman, M., Saidur R., Rahim N. A., Effect of Heat-Generating Solid Body on Mixed Convection Flow in a Ventilated Cavity, Heat Transfer Engineering, 34(15), 12491261 (2013). https://doi.org/10.1080/01457632.20 13.730919

Raji, A., Hasnaoui, M., Mixed Convection Heat Transfer in a Rectangular Cavity Ventilated and Heated from the Side, Numerical Heat Transfer, Part A: Applications, 43, 533-548 (1998). https:// doi.org/10.1080/10407789808913953

Shuja, S. Z., Yilbas, B. S., Jamal, A., Entropy Generation in Flow Field Subjected to a Porous Block in a Vertical Channel, Transport Porous Med., 72, 179-197 (2008). https://doi.org/10.1007/ s11242-007-9143-2

Van Santen, H., Kleijn, C. R., Van Den Akker, H. E. A., On Multiple Stability of Mixed-Convection Flows in a Chemical Vapor Deposition Reactor, International Journal of Heat and Mass Transfer, 44(3), 659-672 (2001). https://doi.org/10.1016/ S0017-9310(00)00121-6

Volker, J., Ellen, S., Finite Element Methods for Time-Dependent Convection-Diffusion-Reaction Equations with Small Diffusion, Computer Methods in Applied Mechanics Engineering, 198, 474-494 (2008). https://doi.org/10.1016/j.cma.2008.08.016

Volker, J., Teodora, M., Michael, R., Kai, S., Lutz, T., Andreas, V., Simulation of Population Balance Systems with One Internal Coordinate Using Finite Element Methods, Chemical Engineering Science, 64, 733-741 (2009). https://doi.org/10.1016/j. ces.2008.05.004

Waghode, A. N., Hanspal, N. S., Shigidi, I. M. T. A., Nassehi, V., Hellgardt, K., Computer Modelling and Numerical Analysis of Hydrodynamics and Heat Transfer in Non-porous Catalytic Reactor for the Decomposition of Ammonia, Chemical Engineering Science, 60(21), 5862-5877 (2005). https://doi.org/10.1016/j.ces.2005.05.019 
Zamora, B., Kaiser, A. S., 3D Effects in Numerical Simulations of Convective Flows in Cubical Open Cavities, International Journal of Thermal Sciences, 77, 172-185 (2014). https://doi.org/10.1016/j. ijthermalsci.2013.11.004

Zamora, B., Kaiser, A. S., Radiative Effects on Turbulent Buoyancy-Driven Airflow in Open Square Cavities, International Journal of Thermal Sciences, 100, 267-283 (2016). https://doi. org/10.1016/j.ijthermalsci.2015.10.002
Zehtabiyan-Rezaie, N., Mirzaei, M., Saffar-Avval, M., Numerical Investigation of Magnetic Field Effect on Heat Transfer and Entropy Generation in Channel; New Approach for Fluid and Length Scale Selection, Heat Transfer Engineering, 38(13), 1222-1232 (2017). https://doi.org/10.1080/01457632.2016.1239961

Zienkiewicz, O. C., Taylor, R. L., Nithiarasu, P., The finite element method for fluid dynamics, Seventh edition, Butterworth-Heinemann publication, USA, (2014). https://doi.org/10.1016/B978-1-85617-635-4.00014-5 